\title{
Jocelyn N. Hillgarth, medievalista, hispanista e historiador de la Iglesia
}

\author{
Jocelyn N. Hillgarth, medievalist, hispanist \\ and historian of the Church
}

Rafael RAMIS BARCELÓ

Departamento de Derecho Público

Universitat de les IIles Balears, IEHM

Universidad Bernardo O'Higgins (Chile)

https://orcid.org/000o-0003-1756-6695

r.ramis@uib.es

Abstract: This essay reviews the fruitful research career of Professor Jocelyn N. Hillgarth (1929-2020), from four main areas: 1) Visigothic Hispania and the spread of early medieval Christianity; 2) Politics, Church and Culture in the Crown of Aragon; 3) Ramon Llull and Lullism; and 4) the cultural and ecclesiastical History of the Kingdom of Mallorca.

Keywords: Jocelyn N. Hillgarth; Medievalism; Hispanism; Roman Catholic Church; Krown of Aragon.
Resumen: Este ensayo repasa la fecunda trayectoria investigadora del profesor Jocelyn N. Hillgarth (19292020), a partir de cuatro grandes ámbitos: 1) la Hispania visigótica y la difusión del cristianismo altomedieval; 2) la política, la Iglesia y la cultura en la Corona de Aragón; 3) Ramon Llull y el lulismo; y 4) la historia cultural y eclesiástica del Reino de Mallorca.

Palabras clave: Jocelyn N. Hillgarth; medievalismo; hispanismo; Iglesia católica; Corona de Aragón.

El 12 de abril de 2020 falleció en Londres el profesor Jocelyn N. Hillgarth, prestigioso medievalista, hispanista, historiador de la Iglesia y miembro que fue del Consejo asesor de Anuario de Historia de la Iglesia entre 1992 y 2008. Sobrevivió pocos meses a su esposa, Nina Pantaleoni (1928-2019), con quien había contraído matrimonio en 1966.

En este escrito pretendemos homenajear su poliédrica figura intelectual, fecunda en diversos campos. Subrayamos, de entrada, que, si para su discípulo Mark D. Meyerson ${ }^{1}$ la obra de Hillgarth puede dividirse en tres grandes áreas,

1 Véase Mark D. MeYERSOn, El profesor Focelyn N. Hillgarth, en Anuario de Estudios Medievales, 26 (1996), pp. 489-501, especialmente p. 490. Véase asimismo la semblanza y el conjunto de estudios que le dedicaron sus discípulos: Thomas E. BURMAN, Mark D. MEYERSON y Leah SHOPKOW (eds.), Religion, Text, and Society in Medieval Spain and Northern Europe: Essays in honor of 7.N. Hillgarth, Toronto, 2002. 
nosotros consideramos que su atención al lulismo debe desgajarse del conjunto de la historia de Mallorca, pues sus contribuciones a dicho campo son tan relevantes que merecen un apartado independiente. Dividimos, por lo tanto, su legado intelectual en cuatro grandes bloques: 1) la Hispania visigótica y su relación con el cristianismo altomedieval; 2) la historia política, eclesial y cultural de la Corona de Aragón; 3) Ramon Llull y el lulismo; y 4) la historia cultural y eclesiástica del Reino de Mallorca.

A fin de comprender mejor su bibliografía, proporcionaremos -antes de entrar en materia- unas breves pinceladas prosopográficas, para entender de qué forma su biografía determinó sus intereses académicos.

\section{NOTAS BIOGRÁFICAS}

Entre los ascendientes del profesor Hillgarth encontramos diversos personajes notables. Su padre fue el Capitán de corbeta Allan H. Hillgarth (18991978), hijo de dos prestigiosos médicos², y su madre, Mary Sidney Katharine Almina (Gardner) Hillgarth (1896-1994), era hija del parlamentario Lord Herbert Colstoun Gardner (1846-1921), primer Baron Burghclere, y de Lady Winifred Anne Henrietta Christine (Herbert) Gardner (1864-1933). Esta última era hija de Henry Herbert, cuarto Conde de Carnarvon. Una hermana de la madre de Jocelyn fue la primera esposa del novelista Evelyn Waugh ${ }^{3}$.

Aunque nació en Londres el 22 de septiembre de 1929, a los pocos meses se trasladó junto con su familia a Mallorca. Sus padres se habían casado en enero de dicho año, cuando su madre obtuvo el divorcio de Geoffrey Hope-Morley, el segundo Baron Hollenden, de quien tenía ya dos hijas.

La figura de su padre ha sido muy estudiada ${ }^{4}$, pues fue vicecónsul y cónsul británico durante la Guerra Civil. Adquirió en 1930 la emblemática finca de Son Torrella $a^{5}$, en el municipio mallorquín de Santa Maria y, desde allí, tejió una importante red de contactos. En dicho predio acogió a diversas personalidades, entre ellas, a Winston Churchill, con quien entabló una firme amistad, y quien

2 Peter DaY, Franco's Friends: How British Intelligence Helped Bring Franco to Power in Spain, London, 2011, cap. 5.

3 John Howard Wilson, Evelyn Waugh, A literary biography, 1924-1966, London, 1996, pp. 39-41.

4 Josep MASsot i MunTaner, El cònsol Alan Hillgarth i les Illes Balears (1936-1939), Barcelona, 1995.

5 Jocelyn N. HillgarTh, Els Torrella de Santa Maria, en Memòries de la Reial Acadèmia Mallorquina d'Estudis Genealògics, Heràldics i Històrics, 11 (2001), pp. 45-57. 
le promovió, acabada la contienda civil, a Agregado de la Embajada británica en Madrid, desde donde Allan colaboró en varias operaciones durante la Segunda Guerra Mundial. Luego, dejó España, se divorció de su esposa, contrajo nuevas nupcias y se retiró a una finca cerca de Tipperary (Irlanda) ${ }^{6}$.

Mary Hillgarth, autora de unas memorias llenas de recuerdos y observaciones interesantes, editadas por Jocelyn ${ }^{7}$, conservó el apellido de su esposo, con el que firmó una traducción al inglés de la Crónica de Pedro el Cerimonioso, en dos volúmenes, prologada y anotada por su hijo ${ }^{8}$.

La infancia de Jocelyn transcurrió en Mallorca y, tras haber estudiado en el Colegio de Montesión, en Palma, se desplazó a Cambridge para continuar su formación. Allí consiguió los títulos de Bachelor of Arts (1950), el de Master of Arts (1954) y el grado de Doctor (1957), al defender la tesis A Critical Edition of the 'Prognosticum futuri saeculi' of St. Fulian of Toledo. Su carrera investigadora empezó como «Senior Research Fellow» en el Warburg Institute de Londres (19591962); y luego, ya en los EEUU, continuó como investigador en la Universidad de Princeton (1963-1964). Posteriormente, empezó su singladura docente: dictó cursos de historia medieval en las Univesidades de Texas (1964-1965), Harvard (1965-1970), en el Boston College (1970-1977) y, finalmente, en la Universidad de Toronto (1977-1995), en la que trabajó hasta su jubilación. En Toronto se desempeñó como Catedrático de Historia medieval y como «Senior Fellow» del Pontifical Institute of Medieval Studies. Hay que recordar que fue también investigador visitante en las Universidades de Cambridge y Hebrea de Jerusalén, y que dictó en 1991 la «Étienne Gilson Lecture» , en el Pontifical Institute of Mediaeval Studies.

Asimismo, aceptó diversas membresías honoríficas y recibió numerosos premios y distinciones: Fobn Guggenheim Memorial Fellow (1968-1969), Magister de la Maioricensis Schola Lullistica (1969), Miembro correspondiente de la Reial Academia de Bones Lletres de Barcelona (1974), Fellow del American Council of Learned Societies (1976-1977), Miembro correspondiente de la Real Academia de la Historia (1977), Fellow de la Medieval Academy of America (1979), Fellow de la Society

6 Josep Massot i MunTaner, El cònsol Alan Hillgarth... [ver nota 4], p. 13.

7 Mary HillgarTH, A private life, Son Torrella, 1984.

8 Chronicle - Pere III of Catalonia (Pedro IV of Aragon), trad. Mary HillgarTh; introd. y notas de J.N. HiLlgarTh, Toronto, 1980.

9 Jocelyn N. HillgarTh, Who Read Thomas Aquinas?, en The Gilson Lectures on Thomas Aquinas, Toronto, 2008, pp. 46-73. Se trata de una lectura muy sugerente acerca de la difusión de los escritos del Aquinate inmediatamente después de su muerte. 
of Antiquaries de Londres (1986), Connaught Senior Fellow de la Universidad de Toronto (1987-1988), Premio Serra d'Or (1993), Premio Catalonia del Institut d'Estudis Catalans (1994), Charles Homer Haskins Medal de la Medieval Academy of America (1995), Miembro de The British Academy (1995)...

Cabe subrayar que el profesor Hillgarth escribió su obra en inglés, castellano y catalán. Algunos de sus trabajos tienen versiones en dos de estas lenguas. Desde su jubilación, repartía su tiempo entre los EE.UU., Londres y Mallorca, y siguió investigando y publicando -hasta concluir la primera década del siglo XXIsobre los temas a los que había dedicado buena parte de su vida académica activa, sobre los que pasamos a continuación.

\section{LA HISPANIA VISIGÓTICA Y EL CRISTIANISMO ALTOMEDIEVAL}

A los ochenta años, en 2009, Hillgarth publicó un ensayo en el que evaluaba la pervivencia del mito visigótico en la historia hispana ${ }^{10}$. Desde la atalaya de la senectud, repasó el interés por lo visigodo en España desde los primeros reyes astures hasta el general Franco. A su juicio, el visigotismo, con altibajos, se impuso más como un mito que como una realidad histórica. Hillgarth, apegado a las fuentes, mostró cierto escepticismo sobre el carácter «gótico» de aquella monarquía hispánica formada a raíz de la descomposición del bajo Imperio Romano de Occidente. Lo «gótico» fue más bien accidental, frente a la continuidad de lo hispanorromano. Para el medievalista inglés, lo sustancial era la herencia romana y católica, verdadero elemento unificador de aquella monarquía hispana, que se perdió con Don Rodrigo. Hillgarth no negó la existencia de una minoría goda que confería una gran originalidad a aquella sociedad ${ }^{11}$, y que adoptó formas culturales singulares. Sin embargo, la Monarquía de Toledo no era tan «gótica» como ha pretendido la historiografía, y mucho menos lo fueron los Reinos cristianos forjados durante la Reconquista.

Estas ideas de Hillgarth subrayan que todos los reyes posteriores de España quisieron retomar el mito de los visigodos para legitimar su poder, si bien no existió una Hispania plenamente visigótica, sino una Hispania católica, con al-

10 Jocelyn N. HillgarTH, The Visigoths in History and Legend, Toronto, 2009, p. XI. «While this is true of many periods of history, it is certainly true of the Visigothic kings who were enthusiastically adopted as models by one age after another, from the rudimentary kingdom of Asturias in the ninth century to the world-monarchy of Spain under the Catholic Kings and the Habsburgs».

11 Ibid., p. 16. 
gunas singularidades «góticas». Este enfoque parte de la lectura eminentemente eclesial del período, que tuvo su punto de partida en su tesis doctoral, la edición crítica del Prognosticum de San Julián de Toledo, escrito escatológico sobre el que reflexionó nuevamente al final de su carrera académica ${ }^{12}$. Aunque ya existían ediciones de dicha obra, la tarea de Hillgarth fue bucear en las diferentes bibliotecas europeas a fin de lograr un catálogo completo de los manuscritos y preparar la ansiada edición crítica ${ }^{13}$.

Tal labor le permitió conocer diversas bibliotecas y trabajar algunas fuentes acerca de la apologética cristiana y la difusión del cristianismo en Irlanda. Algunos de sus estudios tempranos mostraron la influencia del cristianismo visigodo y de los Concilios de Toledo en tierras irlandesas ${ }^{14} \mathrm{y}$ revelaron que uno de los resultados del renacimiento isidoriano fue la transmisión (mediante los monasterios bretones de Galicia) del saber clásico y patrístico a Irlanda: desde allí los escritos de San Isidoro se convirtieron en la base del Renacimiento de Northumbria.

Estos trabajos fueron recopilados junto con otros sobre historiografía visigótica en Visigothic Spain, Byzantium and the Irish ${ }^{15}$. Esta obra no solamente recogía las reflexiones anteriores acerca de la historiografía y la religiosidad popular en el Reino Visigodo, sino los vínculos de la Monarquía y la Iglesia visigoda con Bizancio. De acuerdo con la lectura de Hillgarth, la monarquía visigoda tenía muchos más vínculos con el mundo bizantino que con los demás reinos godos de Europa. Es más, la romanidad -que vindicaba Hillgarth por encima de otros elementos «góticos»- se debía, muy especialmente, a esa afinidad espiritual y política que tuvo la Monarquía Visigótica con el Imperio Bizantino.

No se trataba de una simple convergencia religiosa, sino también política. En una concepción quizás algo polémica, y comentando a Thompson ${ }^{16}$, Hillgarth sostuvo que la monarquía visigoda no solo se configuró política, militar y culturalmente a imagen del Imperio Romano de Oriente, sino que la alianza de los

12 Jocelyn N. HillgarTh, Eschatological and Political Concepts in the Seventh Century, en Jean FonTaINE y Jocelyn N. HillgarTh (eds.), The Seventh Century, Change and Continuity, London, 1992, pp. 212-235.

13 Jocelyn N. Hillgarth, El 'Prognosticum futuri saeculi' de San Fulián de Toledo, en Analecta Sacra Tarraconensia, 30 (1957), pp. 5-61, especialmente 44-61. Hillgarth publicó la edición de esta obra, con otras del mismo autor, en Sancti Iuliani Toletani sedis episcopi opera. Pars I, Corpus Christianorum, series latina 115, Turnhout, 1976.

14 Jocelyn N. HillgarTH, Visigothic Spain and Early Christian Ireland, en Proceedings of the Royal Irish Academy, 62 (1962), pp. 167-194.

15 Jocelyn N. HillgarTH, Visigothic Spain, Byzantium and the Irish, London, 1985.

16 E. A. ThOmpson, The Conversion of the Visigoths to Catholicism, en Nottingham Mediaeval Studies, 4 (1960), pp. 4-35. 
católicos hispanos con Constantinopla fue fundamental para la conversión de los reyes visigodos desde el arrianismo al catolicismo ${ }^{17}$. De hecho, para Hillgarth, la fórmula religiosa de la rebelión de Hermenegildo solo cobraba sentido si se tenía en cuenta la presencia bizantina en la Península ${ }^{18}$. Toda la cultura visigótica católica -incluyendo a los personajes más destacados, como San Isidoro ${ }^{19}$ o San Julián de Toledo- no puede comprenderse sin sus fortísimas ataduras con Bizancio, que empezaron a desligarse progresivamente después del año 589.

Frente a los historiadores germanistas, Hillgarth desarrolló, sobre todo, la vertiente romano-bizantina y católica, en un sentido ideológico (por ejemplo, en la acuñación de moneda, o la propaganda de todo tipo), aunque también en una orientación político-militar. De ahí que el medievalista inglés llegara a considerar la Monarquía visigótica como un estado «sub-bizantino» ${ }^{20}$, es decir, una suerte de protectorado bizantino, que coexistía con las posesiones del Imperio de Oriente en el Levante y Sur peninsular, las cuales -a su juicio- tenían para Constantinopla la misma consideración militar y estratégica que los territorios del Norte de África.

En todo caso, el análisis de la conversión de los reyes visigodos al catolicismo permitió a Hillgarth un estudio de más amplios vuelos sobre el tránsito del paganismo al cristianismo en Europa occidental. La primera edición del texto fue titulada The Conversion of Western Europe, 350-75021, publicada en 1969, que fue ampliada -en 1986- con el título Christianity and Paganism 350-750. The conversion of Western Europe, 350-75022. Se trata de una colección de documentos traducidos al inglés, sobre el tránsito del paganismo al cristianismo en la Europa occidental de finales de la Antigüedad y en los primeros siglos de la Edad Media. Por un lado, la obra muestra la transición del mundo romano al mundo bárbaro durante la descomposición del bajo Imperio (la configuración de la Iglesia romana, las relaciones entre la Iglesia y

17 Jocelyn N. HillgarTh, La Conversión de los Visigodos: notas críticas, en Analecta Sacra Tarraconensia, 34 (1961), pp. 21-46.

18 Jocelyn N. HillgarTh, El Concilio III de Toledo y Bizancio, en Concilio III de Toledo, XIV Centenario 589-1989, Toledo, 1991, pp. 297-306.

19 El autor publicó tres eleborados trabajos de bibliografía isidoriana: Jocelyn N. HILlgarTH, The Position of Isidorian Studies, a Critical Review of the Literature since 1935, en Isidoriana, 1961, pp. 1174; Jocelyn N. HillgarTh, The Position of Isidorian Studies, a Critical Review of the Literature 1935-1975, en Studi medievali, 24 (1983), pp. 817-905; Jocelyn N. HILlGARTH, Isidorian Studies, 1976-1985, en Studi medievali, 31 (1991), pp. 925-973.

20 Jocelyn N. HiLlgarTH, Visigothic Spain, Byzantium...[ver nota 15], p. 457

21 Jocelyn N. HillgarTH, The Conversion of Western Europe, 350-750, Englewood Cliffs, 1969.

22 Jocelyn N. HillgarTh, Christianity and Paganism 350-750. The conversion of Western Europe, 350 750, Philadelphia, 1986. 
el poder político, las primeras conversiones...). Por otro lado, encontramos algunos textos que explican la introducción del cristianismo en una Europa fragmentada, romana, germánica, católica y pagana. Hillgarth estudió de qué forma toda Europa pasó progresivamente a la obediencia católica, no solo desde el paganismo, sino también desde algunas «herejías». En determinados reinos godos se produjo una ósmosis entre la Iglesia y la nueva monarquía, mientras que en otros se llevó a cabo un lento proceso de cristianización (Irlanda, Galia del Norte, Inglaterra, en buena parte del futuro Imperio Romano Germánico...)

Hillgarth, pese a tratar en dicha obra cuestiones que antes no había frecuentado, destacó las conversiones de Clodoveo y de Recaredo, e insistió en los temas que él ya había analizado previamente (la evangelización de Irlanda o las relaciones entre Bizancio y los visigodos), a los que se deben añadir otros, como, por ejemplo, la reforma litúrgica de Occidente. Como idea general, nuestro autor defendió que el hecho más trascendental ocurrido en la Europa Occidental entre los siglos IV y VIII fue la conversión de los distintos pueblos al cristianismo, unida al desplazamiento del centro neurálgico de Europa desde el Mediterráneo al Norte. Hillgarth subrayó las tensiones entre el cristianismo y las religiones ancestrales, que seguían resultando tan atractivas como las doctrinas de la Iglesia, como mínimo hasta el siglo VII, cuando la jerarquía sustituyó los elementos irracionales paganos por los cristianos ${ }^{23}$.

Por lo general, los estudios de Hillgarth sobre el mundo visigodo -en la misma línea que Jacques Fontaine o José Orlandis- enfatizaron las claves religiosas de aquel período, que el medievalista inglés aplicó luego al Occidente tardorromano y altomedieval, en una lectura histórico-antropológica que ha merecido el favor de los especialistas.

\section{Política, IgLesia Y CUltura EN LA CORONA DE ARAGÓN}

En tanto que conocedor de la realidad hispánica, Hillgarth se dedicó a explicar tanto la génesis de la Monarquía Hispánica, como a analizar la evolución de la Corona de Aragón. En realidad, como estudioso de la Monarquía visigótica, comprendió las particularidades de cada uno de los Reinos medievales hispánicos en relación a su pasado, y logró individualizar algunos de los mayores problemas historiográficos de la Corona de Aragón.

23 Ibid., pp. 1-5. 
Sin duda, el trabajo que le reportó mayor notoriedad, en oposición a Lee Shneidman ${ }^{24}$ y, en menor medida, a Vilar y a Soldevila, fue un breve ensayo sobre el problema de un Imperio mediterráneo catalán desde 1229 hasta $1327^{25}$. En realidad, según Hillgarth, la historiografía catalana contemporánea no había sido -con respecto del supuesto «Imperio catalán»- tan hiperbólica como la de los autores no hispanos. Para el profesor londinense, era notoriamente exagerado hablar de un verdadero «Imperio catalán» en el Mediterráneo durante los siglos XIII y XIV. El autor desbrozó el problema analizando la unión personal de los diferentes territorios, y la presencia de dinastías fuertemente emparentadas, aunque sin una política imperial unitaria. Más bien, como detalló a lo largo del opúsculo, se sucedieron contiendas entre los reyes de Aragón, Mallorca y Sicilia. Parece que la propaganda de las Crónicas despistó a los historiadores, quienes creyeron en la épica de los hechos allí narrados, y los interpretaron desde la historia del siglo $\mathrm{XV}^{26}$. Para Hillgarth, la unificación de los dominios mediterráneos de la Corona de Aragón y su expansión se debió más a un cúmulo de factores (entre ellos, los errores de los franceses) que a una verdadera política imperial. Con todo, el autor no negó una creciente pujanza económica, que coadyuvó a la hegemonía mediterránea catalano-aragonesa del siglo XV.

Esta cuestión fue tratada con mayor amplitud en su obra The Spanish Kingdoms, 1250-1516, en dos volúmenes ${ }^{27}$, que ha sido durante muchas décadas un manual muy socorrido para el estudio de la Baja Edad Media hispana en las universidades británicas y norteamericanas. La síntesis de Hillgarth resulta, todavía hoy, muy clara: supo recoger lo mejor de la historiografía hispana y extranjera, así como los frutos de su contacto continuado con muchas fuentes primarias, para ponerlo al servicio del público anglosajón.

Naturalmente, en la obra aparecían los intereses investigadores de Hillgarth, como la cultura libresca o el lulismo, como puede verse en el primer tomo, intitu-

24 Jerome Lee SHnEIDman, The Rise of the Aragonese-Catalan Empire: 1200-1350, New York, 1970.

25 Jocelyn N. HillgarTH, The Problem of a Catalan Mediterranean Empire, 1229-1327, Suplemento de la English Historical Review, London, Longmans, 1975.

26 Ibid., p. 52. «Can one speak of a Catalan 'empire' or of Catalan 'imperialism' in the thirteenth and fourteenth centuries? One can certainly find extravagantly arrogant statements. [...] Ruggiero di Loria, in a well-known boast, could tell the count of Foix that no fish dared cross the sea unless it bore the arms of Aragon. The count smiled. Modern historians might well have followed his example. It seems unwise to use the nineteenth-century term 'imperialism' to describe a very different age. To suppose an attempt to make the Mediterranean 'a Catalan lake' reminds one rather of Benito Mussolini than of the cautious Jaume II, with his plans for a limited hegemony in the western Mediterranean. The idea of a Catalan 'empire' seems even more disleading».

27 Jocelyn N. HillgarTH, The Spanish Kingdoms, 1250-1516, 2 vols., Oxford, 1976-1978. 
lado Precarious Balance. Mostró cautela hacia las tesis económicas de Vicens Vives y trazó una historia en la que la convivencia de las tres religiones no resultaba tan armónica como en la obra de Américo Castro. Hillgarth enfatizó no solamente la complejidad política de la formación de las Coronas de Castilla y de Aragón, sino también el papel de las minorías judía y musulmana, tratadas con especial sensibilidad.

En el segundo volumen, titulado 1410-1516. Castilian Hegemony, presentó una síntesis vívida del siglo XV, quizás especialmente pintoresca en el tratamiento de algunos temas, como el de la religión. En este sentido, trató la cuestión desde una perspectiva más bien anecdótica, como un fresco de clérigos mundanos, faltos de una verdadera reforma espiritual. Puede decirse que Hillgarth actuó con cierto pudor -«con corrección eclesial», diríamos hoy-, prefiriendo una caricatura de los vicios de la Iglesia, sazonada con mil y un ejemplos, antes que un análisis profundo del estamento eclesiástico, que tal vez hubiera arrojado unas consecuencias más deplorables. En todo caso, las extensas semblanzas de Pero López de Ayala y de Francesc Eiximenis ${ }^{28}$ permiten conocer mejor el enfoque del autor.

Como estudioso de la Corona de Aragón, Hillgarth se adelantó a buena parte de la historiografía de los últimos cuarenta años, al defender -con Mario del Treppo $^{29}$ - que la pujanza del Principado de Cataluña se mantuvo hasta la Guerra Civil de los años 1462-1472. Tal circunstancia frenó la expansión catalana y la situó en una posición de debilidad frente a Castilla. No hay duda de que Hillgarth tenía una simpatía indisimulada hacia la Corona de Aragón y que, en su obra, no dejó de remarcar los «errores» de los sucesivos monarcas trastámaras, especialmente de Alfonso el Magnánimo y de Juan II.

Más allá de estas síntesis de temas generales de la Corona de Aragón, Hillgarth, como historiador de la Iglesia, escribió, junto a Giulio Silano, sendos trabajos sobre la Diócesis de Barcelona en el siglo XIV. Uno es la transcripción de las Notule Communium del período comprendido entre 1345 y $1348^{30}$, época de auge de la Peste Negra. En dicha obra -complementada luego por la tesis doctoral de Josep Baucells sobre los años anteriores ${ }^{31}$ - aparecen los más variados asuntos pastorales, jurídicos y económicos del obispado barcelonés. El otro es la edición crítica del

28 Ibid., vol. 2, 1410-1516. Castilian Hegemony, pp. 206-212.

29 Ibid., pp. 10, 266.

30 Jocelyn N. HillgarTh y Giulio Silano, The Register 'Notule communium' 14 of the Diocese of Barcelona (1345-1348), Toronto, 1983.

31 Josep BAUCELLS I REIG, Vivir en la edad media: Barcelona y su entorno en los siglos XIII y XIV (12001344), 3 vols, Barcelona, 2004. 
Sínodo diocesano de Barcelona de $1354^{32}$. La publicación de tales obras en inglés significó una universalización de estos temas, pues luego dichos estudios fueron citados en múltiples trabajos de síntesis sobre la Cataluña medieval.

Por último, debemos mentar el importante análisis de la Crónica de Pere el Cerimoniós, que -como hemos indicado antes- su madre había traducido, y de la cual hizo un estudio preliminar, traducido luego también al catalán ${ }^{33}$ : en él mostraba la relevancia de esta Crónica no solamente en el contexto catalán, sino en toda la historiografía bajomedieval.

\section{RAMON LLULL Y EL LULISMO}

En tanto que católico y mallorquín de adopción, uno de los intereses más profundos del profesor Hillgarth fue el estudio de Ramon Llull, tema que recorre prácticamente toda su vida académica. Para Trias Mercant,

el lulismo de Hillgarth tiene tres centros de interés. En primer lugar, el descubrimiento y estudio de los fondos documentales que fundamentan la obra luliana y los primeros pasos del lulismo. En segundo lugar, la demostración de continuidad del lulismo francés a partir de la muerte de Llull, explicada a la luz de la historia cultural de su época. Por último, perfila los principales episodios del primer lulismo mallorquín y considera que hasta el siglo XV no aparecen en Mallorca maestros y escuelas lulianas ${ }^{34}$.

Las ideas de Trias, que compartimos, dan fe del horizonte investigador de Hillgarth, situado a caballo entre los siglos XIII y XIV, las centurias en las que vivió el Doctor Iluminado. La preocupación heurística -que ha sido un problema esencial para desentrañar la enmarañada bibliografía luliana- le llevó, en un primer momento, a colaborar con Jesús García Pastor y Lorenzo Pérez Martínez en la confección del catálogo de manuscritos lulianos de la Biblioteca Pública de Palma ${ }^{35}$, que desde entonces es una guía imprescindible de ese riquísimo fondo.

32 Jocelyn N. HillgarTh y Giulio Silano, A Compilation of the Diocesan Synods of Barcelona (1354): critical edition and analysis, en Mediaeval Studies, 46 (1984), pp. 78-157.

33 Jocelyn N. Hillgarth, La Personalitat politica i cultural de Pere III a través de la seva Crònica, en Llengua i literatura, 5 (1992-93), pp. 7-102.

34 Sebastià Trias MerCant, Diccionari d'escriptors lul.listes, Palma-Barcelona, 2009, p. 224. [La traducción es nuestra].

35 Jesús García Pastor, J. N. Hillgarth y Lorenzo PÉRez MartíneZ, Manuscritos lulianos de la Biblioteca Pública de Palma, Barcelona-Palma, 1965. 
Luego veremos que buena parte de los intereses acerca de la teología, el mundo eremítico ${ }^{36}$ y la cultura libresca en la Mallorca bajomedieval y renacentista nacieron al calor del estudio archivístico de la figura de Llull y de las doctrinas lulianas ${ }^{37}$.

Tras estudiar los manuscritos lulianos de la biblioteca del Convento franciscano de Dún Mhuire ${ }^{38}$, en Irlanda, aprovechando el impulso de Francis Yates y de Robert Pring-Mill, dio a las prensas un libro sobre Ramon Llull y el lulismo en Francia durante el siglo XIV ${ }^{39}$. En aquellos momentos, se trató de una contribución fundamental, puesto que estudiaba la relación de Llull tanto con Montpellier (que pertenecía, a la sazón, a la Corona de Mallorca) como con el Rey de Francia.

Es especialmente interesante la postura que sostuvo Hillgarth acerca de la relación de Llull con los movimientos espirituales, con los que -sin dudamantuvo afinidades. Sin embargo, para el profesor inglés, el Doctor Iluminado no participó de las ideas rebeldes ni milenaristas, sino que fue siempre un firme defensor del Papado, y una persona plenamente consciente de la realidad política de su época ${ }^{40}$. La relación de Llull con Arnau de Vilanova, analizada por Hillgarth en esta obra y en otras, reabrió un debate en el que ya habían terciado los hermanos Carreras Artau, Batllori y otros, y que ha continuado hasta la actualidad.

En el libro no solamente se detuvo a analizar las relaciones de Llull con la Universidad de París, sino también su presencia en el Concilio de Vienne, así como sus relaciones con Felipe el Hermoso y los sucesivos papas. Dicha obra iluminó también la pervivencia del lulismo francés, especialmente a través de la

36 Jocelyn N. HillgarTH, Some Notes on Lullian Hermits in Majorca, saec. XIII-XVII, en Studia Monastica, 6 (1964), pp. 299-328.

37 Jocelyn N. HILlgarTH, La teología en Mallorca desde el s. XIII al XVI, en Hispania Christiana, Estudios en honor del Prof. Dr. Fosé Orlandis Rovira, Pamplona, 1988, pp. 513-520. «Entre todos los autores registrados en nuestros documentos, Llull, con 204 ejemplares de sus obras, viene en segundo lugar, después del jurista Bartolo de Saxoferrato (con 220). Estos 204 ejemplares estaban distribuidos entre 83 personas. Dejando aparte los Libros de Horas, breviarios y salterios de que hemos hablado, ninguna obra de ningún autor alcanzó más difusión en Mallorca que las obras de Llull». La cita está está en la p. 518.

38 Jocelyn N. HillgarTH, Manuscritos lulianos de la biblioteca del Convento Franciscano de Dún Mbuire, Killiney, Co. Dublin, Irlanda, en Estudios Lulianos, 10 (1966), pp. 73-79.

39 Jocelyn N. HillgarTH, Ramon Lull and Lullism in Fourteenth-Century France, Oxford, 1971. Posteriormente fue publicado en catalán como Ramon Llull i el naixement del lul.lisme, ed. Albert Soler; trad. Anna Alberni y Joan Santanach, Barcelona, 1998.

40 Véase también Jocelyn N. HillgarTH, Raymond Lulle et l'Utopie, en Estudios lulianos, 25 (198183), pp. 175-185. 
obra de Thomas Le Myésier, cuyo Electorium, «primera síntesis lulista» ${ }^{41}$, fue una vía de penetración y consolidación de las doctrinas lulianas entre el público culto. El lulismo francés, a causa de las condenas por parte de Nicolau Eimeric, se mantuvo en una posición de penumbra, pero siguió difundiéndose en cenáculos eclesiásticos y entre algunos maestros universitarios. De ahí el vigoroso rechazo que suscitó en autores como Jean Gerson, quien prohibió la enseñanza de las doctrinas de Llull en París.

Esta obra sigue siendo de lectura provechosa, si bien los estudios lulianos han modificado sensiblemente algunas de las hipótesis de Hillgarth, especialmente en su aproximación al lulismo de la Corona de Aragón. Tras los trabajos de Josep Perarnau ${ }^{42}$, la cuestión del nacimiento del lulismo en Mallorca fue retomada por Hillgarth en un estudio sobre el lulismo mallorquín del siglo XIV ${ }^{43}$, que a su vez ha sido reexaminado por Gabriel Ensenyat ${ }^{44}$, en el marco de las Jornadas que la Cátedra Ramon Llull de la Universitat de les Illes Balears dedicó a Hillgarth y a Anthony Bonner.

El último libro que Hillgarth preparó sobre Llull es una recopilación de documentos sobre la vida de Ramon Barbaflorida: se trata de un diplomatario luliano que reunía por vez primera una colección de cincuenta y un documentos archivísticos relativos a Llull y a su familia ${ }^{45}$, unos más conocidos que otros. La transcripción de los mismos estuvo acompañada, en su caso, de una acertada traducción al catalán por parte de Lluís Cifuentes. Frente a la Vita coaetanea, gracias al buen hacer de Hillgarth, encontramos los avatares de un hombre de carne y hueso, inmerso en el tráfico jurídico y mercantil de su época, y en permanente contacto con los poderes del momento. Estos documentos redimensionan el relato que Llull dejó de sí mismo ${ }^{46}$,

41 Jocelyn N. Hillgarth, Thomas le Myésier: la primera sintesis lulista, en Estudios Lulianos, 16 (1972), pp. 113-126.

42 Especialmente, Josep PERARNAU I EsPelt, El lul.lisme, de Mallorca a Castella a través de València. Edició de l'Art abreujada de confessió, en Arxiu de Textos Catalans Antics, 4 (1985), pp. 61-172.

43 Jocelyn N. HillgarTH, Els començaments del lul.lisme a Mallorca, en Joan MAS I VIVES, Joan Miralles i Monserrat, Pere Rosselló Bover (ed.), Actes de l'Onzè Col-loqui Internacional de Llengua i Literatura Catalanes. Palma (Mallorca), 8-12 de setembre del 1998, Barcelona, 1998, pp. 21-34.

44 Gabriel EnSENYaT PUJOL, L'activitat lul.liana a la Mallorca del segle XIV: un lul.lisme amagat?, en Maria Isabel RIPOLL y Margalida TORTELLA (eds.), Ramon Llull i el lul.lisme: pensament i llenguatge. Actes de les jornades en homenatge a 7.N. Hillgarth i A. Bonner, Palma-Barcelona, 2012, pp. 161-192.

45 Jocelyn N. HiLlgaRTH, Diplomatari lul.lià: documents relatius a Ramon Llull i a la seva família, trad. L. Cifuentes, Barcelona-Palma, 2001.

46 Jocelyn N. HillgarTH, La vida i la significació de Ramon Llull, en Randa, 2 (1976), pp. 5-43; Jocelyn N. HiLlgarTH, Vida i importància de Ramon Llull en el context del segle XIII, en Anuario de estudios medievales, 26 (1996), pp. 967-978. 
y permiten una enriquecedora lectura comparada entre la recreación literaria de la Vita coaetanea y los prosaicos retazos archivísticos reunidos de forma paciente y provechosa.

\section{LA HISTORIA CULTURAL Y ECLESIÁSTICA DEL REINO DE MALLORCA}

Hillgarth dedicó, tal vez, sus mejores páginas a la historia cultural y eclesial del Reino de Mallorca. Su opera prima fue un escrito a cuatro manos con Gabriel Seguí sobre La «Altercatio» y la basilica paleocristiana de Son Bou de Menorca ${ }^{47}$. La edición de esta pieza de la polémica cristiano-judía de finales de la Antigüedad fue publicada de nuevo en 1999, en la que hizo colación de doce manuscritos. Al no poder reproducir todo el texto original, Hillgarth prescindió esta vez, a diferencia de la edición de 1955, de las variantes meramente ortográficas ${ }^{48}$.

De hecho, los tres temas que vertebran la investigación de Hillgarth en este ámbito son: 1) las relaciones entre cristianos y judíos en la Mallorca antigua y medieval; 2) la Diócesis de Mallorca en la época bajomedieval y 3) la historia del libro en la isla durante la Baja Edad Media y el Renacimiento. Muchos de sus escritos buscan una intersección entre esta tríada de intereses.

Sobre los judíos, más allá de la «Altercatio», publicó algunos breves trabajos sobre las disputationes con los cristianos, y otros relacionados sobre todo con su actividad como posesores de libros, u otros datos heurísticos para entender la fisonomía de esta minoría en la isla ${ }^{49}$. De nuevo, cabe tener presente que el lulismo era, en muchos casos, el motor fundamental de su investigación.

Sobre la Diócesis de Mallorca, junto con los trabajos sobre el obispo Antoni des Collell ${ }^{50}$, cabe tener en cuenta, sobre todo, la edición -junto con Juan Rosselló- del Liber communis Curiae de la Diócesis de Mallorca (1364-1374) ${ }^{51}$,

47 Gabriel Seguí y Jocelyn N. HillgarTh, La «Altercatio» y la basilica paleocristiana de Son Bou de Menorca, Palma, Sociedad Arqueológica Luliana, 1955 (extracto de Boletín de la Sociedad Arqueológica Luliana, 31, 1954, pp. 1-60).

48 Altercatio Ecclesiae et Synagogae, cura et studio J. N. HiLlgarTH, «Corpus Christianorum. Series latina, LXIX A», Turnhout, 1999, pp. 1-53.

49 Jocelyn N. HillgarTH, The Disputation of Majorca (1286): two new editions, en Euphrosyne, 22 (1994), pp. 403-413; Jocelyn N. HiLlgarTH, Sources for the History of the Fews of Majorca, en Traditio, 50 (1995), pp. 334-341.

50 Jocelyn N. HillgarTH, Inventario de los bienes de Antoni des Collell, obispo de Mallorca (1349-1363), en Boletín de la Sociedad Arqueológica Luliana, 31 (1953-60), pp. 504-554.

51 Jocelyn N. HillgarTH y Juan ROSSElló LliTERAS, The «Liber communis» of the Diocese of Majorca (1364-1374), Montréal, 1989. 
el único ejemplar superviviente de esta época en Mallorca, correspondiente al pontificado de Antoni Galiana. Como puede suponerse, Hillgarth se dedicó a la labor de comparación con el registro que había editado -con Giulio Silano- de la Diócesis de Barcelona. En este caso, las materias pastorales, jurisdiccionales, financieras... eran menos complejas que las recogidas en el texto de diócesis de la Ciudad Condal.

Desde luego, los autores se hacían eco de cuestiones que no tenían ningún paralelo en la documentación barcelonesa, y buscaron resaltar la originalidad de la fuente estudiada, al tiempo que indicaron, por ejemplo, que Ponç de Gualba, creador del archivo episcopal de Barcelona y de las primeras series de registros episcopales, fue -casi seguro- de origen mallorquín ${ }^{52}$. Cabe resaltar que, si en la documentación barcelonesa se palpaban directamente los estragos de la peste negra, en el caso mallorquín se podían extraer sus consecuencias, no solamente en el ámbito eclesiástico, sino también en el económico, demográfico...

Todos sus estudios sobre la historia del libro desembocaron en la que, con toda probabilidad, es su opus magnum: Readers and Books in Majorca, 1229-155053. Se trata de un trabajo excepcional, que se divide en dos volúmenes, de paginación continua. La obra inicia con una iluminadora y detallada introducción de conjunto (pp. 1-103) a los 916 inventarios de libros editados, que dan fe de la amplia gama de documentos archivísticos consultados por el autor en la propia Mallorca, Barcelona, Madrid y en la Abadía de Montserrat.

La segunda parte de la obra contiene los catálogos de bibliotecas (pp. 305853), con índices detallados (pp. 857-1018). La obra recoge las bibliotecas de la Catedral, de las comunidades religiosas (agustinos, carmelitas, cistercienses, dominicos, franciscanos), de las parroquias, de particulares, así como colecciones profesionales (juristas, notarios, médicos, mercaderes...) y humanistas de la época. No hay duda de que uno de los mayores intereses era aquilatar el papel del lulismo en Mallorca ${ }^{54}$, contemplada como un centro intelectual, pues se detallan no pocas rarezas (por ejemplo, ciertos ejemplares en bibliotecas de médicos judíos), y dan fe de una rica circulación libresca. En efecto, Hillgarth indicó que la isla era, a la sazón, «una encrucijada no sólo de mercancías, sino también de ideas $»^{55}$, y fue capaz de comprender con gran agudeza la complejidad de una

52 Ibid., p. 11.

53 Jocelyn N. HillgarTH, Readers and Books in Majorca, 1229-1550, Paris, 1991, 2 vols.

54 Ibid., pp. 191-234.

55 Jocelyn N. HiLlgarTH, Mallorca como centro intelectual, 1229-1550, en Anuario de estudios medievales, 19 (1989), 205-11. La cita está en la p. 205. 
sociedad como la mallorquina, que gozaba en aquel momento de una variedad social extraordinaria. Las categorías interpretativas de Readers and books, elaboradas con gran sutileza, están destinadas a perdurar no solamente para el estudio de la historia cultural de Mallorca, sino como modelo de análisis en la historia cultural bajomedieval y renacentista.

\section{CONCLUSIONES}

Hillgarth proporcionó una particular hermenéutica del mundo hispánico en sus diversas versiones. Si muchos hispanistas europeos han sido personas con antepasados españoles, el medievalista inglés tuvo en Mallorca su segunda casa y, en ella, se crió. Si damos fe al resabio popular que afirma que uno es de donde ha cursado el bachillerato, Hillgarth era casi español, y por lo tanto, su visión de lo hispano no era algo distante, sino cercano: un inglés que nunca dejó de serlo, y un mallorquín adoptivo, que jamás renegó de sus vínculos con la isla.

Aunque la mayor parte de su obra esté centrada en la Edad Media, hizo -especialmente en su senectud-algunas incursiones en la época moderna, como The Mirror of Spain, 1500-170056, en la cual profundizó en la visión que los otros pueblos europeos tuvieron de la España de los Austrias. No en vano, como escribió el autor, «as compared with other european lands, Spain has proved a hard country for foreigners to understand $»^{57}$.

El «visigotismo» -en su interpretación- fue más un mito que una realidad: prevaleció lo romano y lo católico, lo verdaderamente sustancial, frente a lo germánico, meramente accidental. Su análisis del Reino Visigodo desde Bizancio, muestra la singularidad de esta Monarquía, prácticamente infeudada al Emperador de Oriente. El estudio comparado de la descomposición del Imperio de Occidente y la entrada de los pueblos bárbaros le permitió entender las diferentes fases en la absorción cultural del cristianismo en los distintos puntos de Europa. En este sentido, el caso hispano fue distinto, no solo por su relación con Bizancio, sino también por la presencia judía y, posteriormente, por la conquista islámica.

La «Reconquista» fomentó el mito de lo «visigodo», aunque - para Hillgarth- lo importante era lo romano y lo católico. Cabe subrayar que el hispanismo de Hillgarth se fraguó desde una isla muy apartada del centro neurálgico de Es-

56 Jocelyn N. HillgarTh, The Mirror of Spain, 1500-1700: The Formation of a Myth, Michigan, 2000.

57 Ibid., p. 3. 
paña. Esa visión periférica le dotó de una hermenéutica mucho más refinada y distante de lo hispano, al tiempo que le familiarizó con la cultura de la Corona de Aragón. Como medievalista, observó sabiamente que la cristiandad hispana (desde el siglo VII), con las Coronas de Castilla y Aragón, respiró a dos pulmones, ambos herederos, ciertamente, de las cenizas romanas de la monarquía visigótica, y que mantuvieron conexiones muy diferentes con Italia.

La Corona de Castilla -como subrayó en The Spanish Kingdoms- estuvo más encerrada en sí misma, y sostuvo con mayor tesón, quizás, algunos elementos germánicos. La Corona de Aragón permaneció vencida hacia el Mediterráneo, de modo que no puede estudiarse sino desde la perspectiva del Mare Nostrum (Francia, Italia y el Norte de África), y en una dimensión mucho más latina.

El entrecruzamiento de todos estos aspectos puede verse en Mallorca, solar patrio de Ramon Llull, y punto de encuentro de diferentes culturas. El estudio del lulismo permitió a Hillgarth el análisis de un fenómeno internacional, considerado también desde diversas perspectivas. Readers and Books in Majorca es un extraordinario ejemplo de cómo la historia local puede ser luz y norte para la historia cultural europea.

En la obra de Hillgarth encontramos, en fin, una perspectiva de longue durée. Desde la caída del Imperio Romano de Occidente hasta finales del Medioevo, en ella se entrecruza la historia religiosa con la historia cultural: la microhistoria, estudiada a conciencia, le permitió construir luego un metarelato sólido, crítico y sutil. Desde el mundo visigótico a Fernando el Católico, Hillgarth recorrió los caminos de la Europa medieval: grandes temas y cuestiones menores fueron objeto de su atención. Su estudio de la historia de Mallorca y de la Corona de Aragón, su interés por la historia religiosa y cultural (desde San Julián de Toledo a Ramon Llull), así como su empeño por desentrañar la historia del cristianismo occidental nos permiten recordarle con afecto y admiración. Hillgarth fue, en definitiva, un gran medievalista, hispanista e historiador de la Iglesia: estos tres calificativos, convergentes y acumulativos, permiten apreciar una trayectoria notable y una obra muy valiosa, destinada -sin duda alguna- a perdurar.

REFERENCIAS BIBLIOGRÁFICAS

\section{Fuentes}

Altercatio Ecclesiae et Synagogae, cura et studio J. N. HILlgarTH, «Corpus Christianorum. Series latina, LXIX A», Turnhout, 1999, pp. 1-53. 
Chronicle - Pere III of Catalonia (Pedro IV of Aragon), trad. Mary Hillgarth; introd. y notas de J. N. Hillgarth, Toronto, 1980.

Sancti Iuliani Toletani sedis episcopi opera. Pars I, Corpus Christianorum, series latina 115, Turnhout, 1976.

\section{Bibliografía}

BAUCELLS I REIG, Josep, Vivir en la edad media: Barcelona y su entorno en los siglos XIII y XIV (1200-1344), 3 vols, Barcelona, 2004.

Burman, Thomas E., Meyerson Mark D. y ShOPKOw, Leah (eds.), Religion, Text, and Society in Medieval Spain and Northern Europe: Essays in bonor of 7.N. Hillgarth, Toronto, 2002.

DAY, Peter, Franco's Friends: How British Intelligence Helped Bring Franco to Power in Spain, London, 2011.

Ensenyat PUjol, Gabriel, L'activitat lul.liana a la Mallorca del segle XIV: un lul.lisme amagat?, en Maria Isabel RIPOLL y Margalida TORTELLA (eds.), Ramon Llull i el lul.lisme: pensament i llenguatge. Actes de les jornades en homenatge a 7.N. Hillgarth i A. Bonner, Palma-Barcelona, 2012, pp. 161-192.

García Pastor, Jesús, Hillgarth, J. N. y PÉrez Martínez, Lorenzo, Manuscritos lulianos de la Biblioteca Pública de Palma, Barcelona-Palma, 1965.

Hillgarth, Jocelyn N., Inventario de los bienes de Antoni des Collell, obispo de Mallorca (1349-1363), en Boletín de la Sociedad Arqueológica Luliana, 31 (1953-60), pp. 504554.

HillgarTh, Jocelyn N., El 'Prognosticum futuri saeculi' de San fulián de Toledo, en Analecta Sacra Tarraconensia, 30 (1957), pp. 5-61.

HiLlgarTH, Jocelyn N., La Conversión de los Visigodos: notas críticas, en Analecta Sacra Tarraconensia, 34 (1961), pp. 21-46.

HillgarTh, Jocelyn N., The Position of Isidorian Studies, a Critical Review of the Literature since 1935, en Isidoriana, 1961, pp. 11-74.

Hillgarth, Jocelyn N., Visigothic Spain and Early Christian Ireland, en Proceedings of the Royal Irish Academy, 62 (1962), pp. 167-194.

HiLlgarTH, Jocelyn N., Some Notes on Lullian Hermits in Majorca, saec. XIII-XVII, en Studia Monastica, 6 (1964), pp. 299-328.

HillgarTh, Jocelyn N., Manuscritos lulianos de la biblioteca del Convento Franciscano de Dún Mbuire, Killiney, Co. Dublin, Irlanda, en Estudios Lulianos, 10 (1966), pp. 73-79.

HiLlgarTH, Jocelyn N., The Conversion of Western Europe, 350-750, Englewood Cliffs, 1969.

HiLlgarTH, Jocelyn N., Ramon Lull and Lullism in Fourteenth-Century France, Oxford, 1971.

HiLlgarTH, Jocelyn N., Thomas le Myésier: la primera sintesis lulista, en Estudios Lulianos, 16 (1972), pp. 113-126. 
HillgarTH, Jocelyn N., The Problem of a Catalan Mediterranean Empire, 1229-1327, Suplemento de la English Historical Review, London, Longmans, 1975.

HillgarTh, Jocelyn N., La vida i la significació de Ramon Llull, en Randa, 2 (1976), pp. $5-43$.

HillgarTH, Jocelyn N., The Spanish Kingdoms, 1250-1516, 2 vols., Oxford, 1976-1978.

HillgarTH, Jocelyn N., Raymond Lulle et l'Utopie, en Estudios lulianos, 25 (1981-83), pp. $175-185$.

HillgaRTH, Jocelyn N., The Position of Isidorian Studies, a Critical Review of the Literature 1935-1975, en Studi medievali, 24 (1983), pp. 817-905.

HillgarTH, Jocelyn N., Visigothic Spain, Byzantium and the Irish, London, 1985.

HillgarTh, Jocelyn N., Christianity and Paganism 350-750. The conversion of Western Europe, 350-750, Philadelphia, 1986.

HILlgarTH, Jocelyn N., La teología en Mallorca desde el s. XIII al XVI, en Hispania Christiana, Estudios en honor del Prof. Dr. Fosé Orlandis Rovira, Pamplona, 1988, pp. 513-520.

HillgarTH, Jocelyn N., Mallorca como centro intelectual, 1229-1550, en Anuario de estudios medievales, 19 (1989), 205-11.

Hillgarth, Jocelyn N., El Concilio III de Toledo y Bizancio, en Concilio III de Toledo, XIV Centenario 589-1989, Toledo, 1991, pp. 297-306.

HiLlgaRTH, Jocelyn N., Readers and Books in Majorca, 1229-1550, Paris, 1991, 2 vols.

HillgarTH, Jocelyn N., Isidorian Studies, 1976-1985, en Studi medievali, 31 (1991), pp. $925-973$.

Hillgarth, Jocelyn N., Eschatological and Political Concepts in the Seventh Century, en Jean Fontaine y Jocelyn N. HillgarTh (eds.), The Seventh Century, Change and Continuity, London, 1992, pp. 212-235.

HillgarTh, Jocelyn N., La Personalitat politica i cultural de Pere III a través de la seva Crònica, en Llengua i literatura, 5 (1992-93), pp. 7-102.

HILlGARTH, The Disputation of Majorca (1286): two new editions, en Euphrosyne, 22 (1994), pp. 403-413.

HillgarTH, Jocelyn N., Sources for the History of the Fews of Majorca, en Traditio, 50 (1995), pp. 334-341.

HillgarTH, Jocelyn N., Vida i importància de Ramon Llull en el context del segle XIII, en Anuario de estudios medievales, 26 (1996), pp. 967-978.

HillgarTH, Jocelyn N., Els començaments del lul.lisme a Mallorca, en Joan MAS I VIves, Joan Miralles i Monserrat, Pere Rosselló Bover (ed.), Actes de l'Onzè Col.loqui Internacional de Llengua i Literatura Catalanes. Palma (Mallorca), 8-12 de setembre del 1998, Barcelona, 1998, pp. 21-34.

HillgarTH, Jocelyn N., Ramon Llull i el naixement del lul.lisme, ed. Albert Soler; trad. Anna Alberni y Joan Santanach, Barcelona, 1998.

HiLlgaRTH, Jocelyn N., The Mirror of Spain, 1500-1700: The Formation of a Myth, Michigan, 2000.

HillgarTh, Jocelyn N., Diplomatari lul-lià: documents relatius a Ramon Llull i a la seva família, trad. L. Cifuentes, Barcelona-Palma, 2001. 
Hillgarth, Jocelyn N., Els Torrella de Santa Maria, en Memòries de la Reial Acadèmia Mallorquina d'Estudis Genealògics, Heràldics i Històrics, 11 (2001), pp. 45-57.

HillgarTh, Jocelyn N., Who Read Thomas Aquinas?, en The Gilson Lectures on Thomas Aquinas, Toronto, 2008, pp. 46-73.

HillgarTH, Jocelyn N. y Juan ROSSELló LliTERAS, The 'Liber communis' of the Diocese of Majorca (1364-1374), Montréal, 1989.

HillgarTH Jocelyn N. y Giulio SILANO, The Register 'Notule communium' 14 of the Diocese of Barcelona (1345-1348), Toronto, 1983.

Hillgarth, Jocelyn N. y Giulio Silano, A Compilation of the Diocesan Synods of Barcelona (1354): critical edition and analysis, en Mediaeval Studies, 46 (1984), pp. 78-157.

HillgarTH, Jocelyn N., The Visigoths in History and Legend, Toronto, 2009.

Hillgarth, Mary, A private life, Son Torrella, 1984.

Massot I MunTaner, Josep, El cònsol Alan Hillgarth i les Illes Balears (1936-1939), Barcelona, 1995.<MEYERSON, Mark D., El profesor Focelyn N. Hillgarth, en Anuario de Estudios Medievales, 26 (1996), pp. 489-501.

PERARNAU i ESPELT, Josep, El lul.lisme, de Mallorca a Castella a través de València. Edició de l'Art abreujada de confessió, en Arxiu de Textos Catalans Antics, 4 (1985), pp. 61-172.

SEGUí, Gabriel y HiLlgaRTH, Jocelyn N., La «Altercatio» y la basilica paleocristiana de Son Bou de Menorca, Palma, Sociedad Arqueológica Luliana, 1955 (extracto de Boletín de la Sociedad Arqueológica Luliana, 31, 1954, pp. 1-60).

SHNEIDMAN, Jerome Lee, The Rise of the Aragonese-Catalan Empire: 1200-1350, New York, 1970.

Thompson, E.A. The Conversion of the Visigoths to Catholicism, en Nottingham Mediaeval Studies, 4 (1960), pp. 4-35.

Trias MERCANT, Sebastià, Diccionari d'escriptors lul.listes, Palma-Barcelona, 2009.

WiLSON, John Howard, Evelyn Waugh, A literary biography, 1924-1966, London, 1996. 
